The author's objective is to transmit the maximum of his experience in this field.

I think this book will be of real value because its structure is well conceived and there is an orderly development of the subject in every chapter. It is not necessary to absorb all the mathematical formulae to understand the important points. The first chapters recall the fundamentals of electron optics, with special emphasis on wave optics and coherence. High-resolution images of periodic and non-periodic specimens - the really important topics - are treated in the fifth and sixth chapters. The reader will find a variety of results, well chosen and explained in full detail; he will certainly acquire the ambition and desire to undertake (or continue) research of this kind. The final chapters are, in effect, a compendium of advice for the more straightforward cases.

In conclusion, this book is a very good one, and unquestionably successful.

\section{Faculté des Sciences}

Université de Reims

Moulin de la Housse

51062 Reims CEDEX

France

\section{Acta Cryst. (1983). A39, 504}

Recent developments in condensed matter physics, Vol. 3: Impurities, excitons, polarons and polaritons. Edited by J. T. Devreese, L. F. Lemmons, V. E. VAN DoRen and J. VAN Royen. Pp. xvi +420 . New York: Plenum, 1982. Price US \$55.00.

This volume contains a convenient grouping of contributed papers given at the first European Physical Society Condensed Matter Division's Conference, held in Antwerp, 9-11 April 1980. It is a very interesting and useful volume, and should be read by all who wish to remain abreast of current research. Some concern might be raised that the shear diversity of topic could dilute the worth of the volume 'spread so thin that...'. But no, and, to the very contrary, categories were so chosen and the breadth was so scaled that the topic sections are as coherent as can be expected. More importantly, the overlap integral between the contents of this volume and the interests of the average solid-state physicist is sufficiently large to recommend that everybody spend some time with it, at the very least. There will undoubtedly be one or two papers that will be of great interest, and one is always happy to learn about new, but related, work.

The content is somewhat wider than the title would suggest and includes, in addition, sections on semiconductors and semimetals, electron structure and dielectric properties.

The section on semiconductors and semimetals includes papers on bismuth, electron transport and associated parameters such as quantum oscillations, impurity scattering, hopping conduction, recombination and luminescence.

The section on impurities and defects contains experimental results obtained using EPR, NMR and Mössbauer spectroscopies, the calculation of ionic and electronic states of impurities in a wide variety of hosts including II-VI compounds and also silicon, exciton defect, exciton-exciton interactions with some interesting theoretical contributions. The electronic-structure section covers experimental and theoretical studies of oxides (including $\mathrm{NiO}$ !), garnets, $4-f$ ions. Dielectric properties include oscillator-strength calculations, non-linear optical properties and the final section, polarons and the experimental evidence for their existence in oxides, includes very interesting theoretical papers using a Brownian-motion approach and path-integral calculations, polaritons in $\mathrm{CuCl}$ and $\mathrm{CuBr}$ and optical bistability.

Having explored this volume, one is highly motivated to investigate the other volumes covering the Conference: Vol. 1, The invited papers, Vol. 2, Metals, disordered systems, and interfaces and Vol. 4, Low dimensionality systems; phase changes and experimental techniques.

\author{
Department of Materials Science and \\ Department of Physics \\ Rensselaer Polytechnic Institute \\ Troy \\ New York 12181 \\ $U S A$
}

R. K. MacCrone

\section{Books Received}

The following books have been received by the Editor. Brief and generally uncritical notices are given of works of marginal crystallographic interest; occasionally a book of fundamental interest is included under this heading because of difficulty in finding a suitable reviewer without great delay.

Structural crystallography in chemistry and biology: benchmark papers in physical chemistry and chemical physics. Vol. 4. Edited by J. P. Glusker. Pp. xx +421 . Hutchinson Ross Publishing Co., distributed by Academic Press, New York, 1981. Price $£ 33.00$, US $\$ 50.00$. A review of this book, by M. F. Perutz, has been published in the February 1983 issue of Acta Crystallographica. Section B, page 139.

Structural studies on molecules of biological interest. Edited by G. Dodson, J. P. Glusker and D. Sayre. Pp. xviii + 610. Oxford: Clarendon Press, 1981. Price £39.00. A review of this book, by $S$. Abrahamsson, has been published in the February 1983 issue of Acta Crystallographica, Section B, page 141 . 